\title{
Peculiarities of The Orientational State of Liquid-Crystal Completely Aromatic Thermotropic Copolymer
}

\author{
V.A. Ryzhov*
}

Ioffe Physicotechnical Institute, Russian Academy Science, 194021 St. Petersburg, Russia

\begin{abstract}
Polarization far-IR spectroscopy has been used to carry out a comparative study of the spectra of granules and fibers of completely aromatic thermotropic liquid-crystal copolymer Vectra A950. The analysis of terahertz polarization IR spectra Vectra A950 showed that in contrast to the mid-IR, dichroic ratio of the absorption bands in the spectra of fibers and granules, substantially different. It is shown that the difference between the value and the orientation order parameter in the samples is determined by and correlated with the intensity of the local dynamics of polymer chains, such as the librational mobility of phenylene and naphthalene rings of the copolymer mesogens. The results suggest the possibility carrying out an independent evaluation of the spectroscopic parameters reflecting the properties of intermolecular interactions in oriented the liquid-crystalline polymers for their low-frequency spectra.
\end{abstract}

Keywords: Liquid-crystal polymers, far-infrared spectroscopy, local dynamics, orientation order.

\section{INTRODUCTION}

Currently, liquid crystal (LC) polymers are among the most studied, from the academic and technical point of view, materials. The structure of these compounds provides a high anisotropy of the electrical and optical properties, as well as the opportunity to control these properties and their molecular architecture make it possible to produce on their basis fibers and films for modern high-tech industry $[1,2]$.

A reliable determination of the orientation order parameter characterizing the anisotropy of the LC polymers (LCPs) is a topical task in this regard. To determine this important parameter NMR, fluorescence, Raman and IR spectroscopy have been used [3, 4]. However, the range covered by these studies of LCP is rather limited. This includes mainly LC polyesters containing flexible fragments in the backbone of the macromolecule, whereas the completely aromatic thermotropic LCP with rigidaromatic mesogens in the main chain, which are of particular interest due to the high thermal stability and elasticity modulus, have been insufficiently studied $[5,6]$.

In addition, as far as we know, the orientation order in the LCP has been determined in the previously unused terahertz range $(v<400 \mathrm{~cm}-1)$, in which the main mechanism responsible for the formation of the IR absorption spectra has a clearly pronounced intermolecular nature and at the same time the mere existence of a liquid crystal state is also due to the

*Address correspondence to this author at the loffe Physicotechnical Institute, Russian Academy Science, 194021 St. Petersburg, Russia;

E-mail: v.ryzhov@mail.ioffe.ru forces of intermolecular interaction [7]. Besides, it is well known that the low-frequency spectra of ordered systems (crystals) on the one hand and disordered systems (liquids, glassy substances), on the other, differ significantly among themselves. Since the LCD is occupied in a certain sense, an intermediate position in a number of these systems, it can be expected that the low-frequency spectra of the LCD will clearly reflect the features of their structure. Finally, studies of lowfrequency spectra of LCs are important from the point of view of a deeper understanding of the nature of these spectra, which is still unclear.

It should be noted that low-frequency IR spectra of LCP studied not enough. Yet now it established that they carry information about the structure and the degree of ordering of the LCP, as well as the dynamics of molecular motion [8].

In this study, we were interested above all the in using the terahertz range to evaluate differences in the molecular mobility and specific features of the orientation state in fibers and granules of completely aromatic thermotropic liquid-crystal copolymer Vectra A950.

LCPs Vectra A950 is typically full-aromatic copolyester and has a rod-like shape. The reinforcement arises from the structure of the LCP molecules and their alignment in the mesophase [9]. Mesomorphic regions with parallel alignment form insitu fibers in the solid state. In blends with other thermoplastics, this provides self-reinforcement [10]. The material can further be reinforced by adding glassfibers. In any case, the specific mechanical properties arise from the fibrillar structure of the LCP. 
Macromolecules of the LCP are so rigid that a segmental motion is impossible in these materials. High molecular orientation in fibers of LCP, which achieved in the spinning process and on the special heat treatment, is lead to the significant structure transformations. For such large-scale rearrangement a specific molecular mobility has to occur. Studies the molecular mobility in fibers and granules of LCP Vectra A950 is the subject of this work. Far-infrared spectroscopy was used for this purpose.

An analysis of the terahertz polarization IR spectra Vectra A950 showed that in contrast to the mid-IR, the dichroic ratio is substantially different in the absorption bands in the far-infrared spectra of fibers and granules. This difference is particularly evident for the absorption bands, characterizing the libration of the phenylene and naphthalene rings of the copolymer at 98 and $127 \mathrm{~cm}^{-1}$, respectively. These data allow us to state that the orientation state of the LCP type Vectra A950 is largely determined by the local dynamics of polymer chains, such as the librational motion of aromatic groups in the thermotropic copolyester.

The results of this study show the efficiency of lowfrequency IR spectroscopy in evaluating the order parameter and specific features of the orientation state of a liquid-crystal polymer.

\section{MATERIALS AND METHODS}

As the object of the study served an industrial grade LCD copolymer Vectra A950, which has the formula:

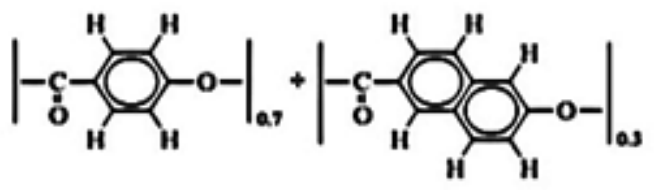

and contains73 mol. \% 4-hydroxybenzoic acid units and 27 mol. \% 2-hydroxy-6-naphthoic acid units. Granules of the copolymer were obtained by molding the starting material purchased from Hoechst-Celanese corp. Its fibers are made in a laboratory by the PTI method [9]. The main feature of the Vectra A950 LCD polymer is that it has in the main chain with two mesogenic groups that are not separated by flexible junctions. This makes copolymer macromolecules stiff and significantly limits the molecular mobility of the main chain [10].

To estimate of the orientation order parameter of the copolymer $S=1 / 2(3<\operatorname{Cos} 2 \Theta>-1)$, where $\Theta$ - is the -angle between the long axis of an individual molecule and the draw direction of the fiber, we used the expression $S=(R-1)\left(R_{0}+2\right) /(R+2)\left(R_{0}-1\right)[11]$, where $\mathrm{R}$ - is the- dichroic ratio, $\mathrm{R}_{0}=2 \operatorname{Cot}^{2} \psi$, a $\psi$ is theangle between the dipole transition moment related to the absorption and the long axis of an individual molecule which in this case is parallel to the stretching direction. The angle $\psi$ for the main components of Vectra A950- benzene rings and C-O-C bonds is not exactly known, but it can be taken, to a first approximation, equal to zero [12], and then $S=(R-1) /$ $(R+2)$. The dichroic ratio $R$ is defined as $R=A_{\|} / A_{\perp}$, where $A_{\|}$and $A \perp$ are the absorbance for the IR light polarized parallel and perpendicular to the director, respectively. The absorbance $A_{\|}$and $A \perp$ are given by the Lambert-Beer law: $A_{\|}=\log _{10}\left(1 / T_{\| I}\right)$ or $A_{\perp}=$ $\log _{10}\left(1 / T_{\perp}\right)$, where $T_{\| I}$ and $T_{\perp}$ are the transmittance for linearly polarized IR light with electric vectors parallel and perpendicular to the director, respectively.

The IR transmission spectra of Vectra A950 were measured with a Perkin-Elmer 577 IR spectrophotometer in the $4000-400 \mathrm{~cm}^{-1}$ spectral range with resolution of $2-4 \mathrm{~cm}^{-1}$ and spectrometer Hitachi FIS-21 in the region $400-20 \mathrm{~cm}^{-1}$ with resolution of $1-2$ $\mathrm{cm}^{-1}$. Polarized spectra were obtained by using wiregrid (1200 grooves $/ \mathrm{mm}$ ) polarizers on PTFE and PE substrates.

The samples used in experiments were prepared as a parallel set of fiber rods end- glued on a framework with dimensions $12 \times 20 \mathrm{~mm}$. The IR transmission spectra Vectra A950 granules were measured by using polyethylene pellets. The pellets were made by mixing a 2- mg sample with $200 \mathrm{mg}$ of spectroscopic grade polyethylene and pressed into pellets using hydraulic press. To take into account the polyethylene absorption, the polyethylene spectrum was used as reference. The sample spectrum was divided by the reference spectrum to nullify the polyethylene absorption.

\section{RESULTS AND DISCUSSION}

The IR-spectra of Vectra A950 in the range of 4000$400 \mathrm{~cm}-1$ are shown in Figure 1. The absorption bands in these spectra were assigned in $[5,13]$. Far- IR spectra of Vectra A950 in the range $480-20 \mathrm{~cm}^{-1}$ are shown in Figure 2. It should be noted that the far- IR spectra of Vectra A950 were measured for the first time. To date only far-IR spectra of low molecular LC have been studied in detail, but they are sufficiently scattered and primarily related to low-molecular 


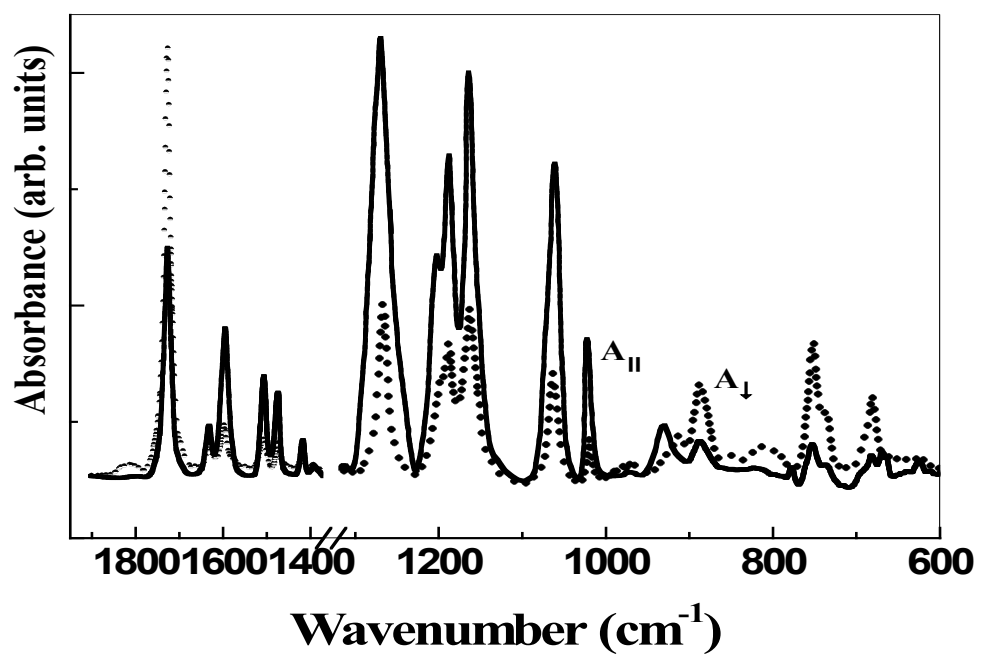

Figure 1: Polarized IR- spectra Vectra A950 recorded for parallel $(A \|)$ and perpendicular $(A \perp)$ arrangements of the $E$ vector to the fiber axis.

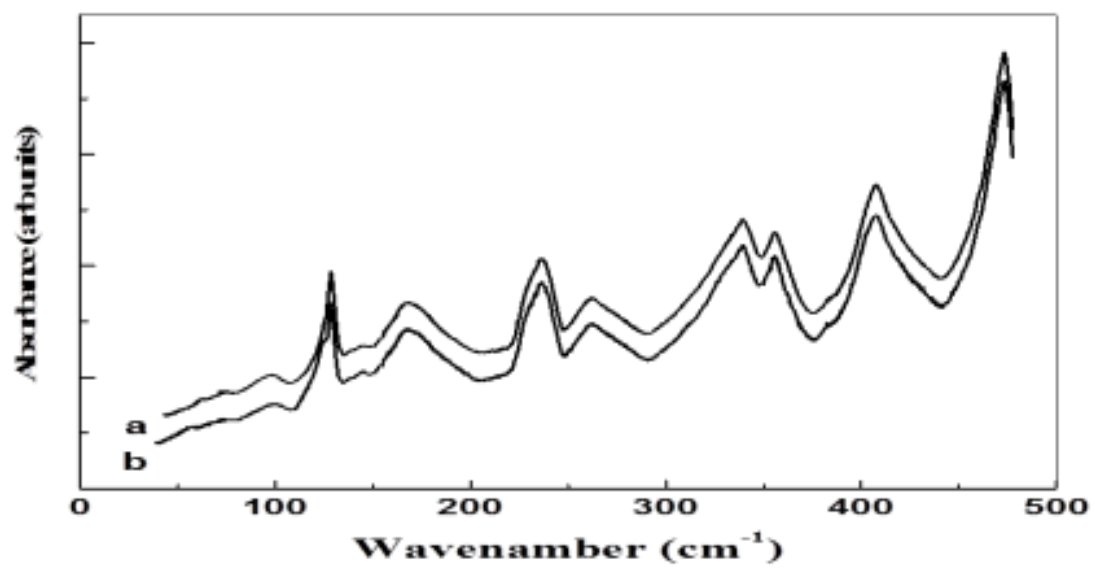

Figure 2: Far-IR spectra Vectra $A 950$ in the range $30-500 \mathrm{~cm}^{-1}$. Spectrum of fibers (a) shifted on the ordinate.

nematics type of MBBA (4-methoxy-4-benzylideneaniline) with benzene rings as a mesogens and alkyl groups as a flexible spacers $[14,15]$.

It was found that the mid-IR spectra of Vectra A950 fibers and granules Vectra A950 are almost identical. This result reflects that fact that short-range intramolecular forces operating in fibers are the same as those in granules. By contrast, vibrational bands of molecules in the low-frequency spectral range, typically below $\sim 300 \mathrm{~cm}^{-1}$, arise not only from intramolecular interactions but also from intermolecular interactions of the molecules. Vibrational spectroscopy in this frequency range is focused on the information concerning both the molecular motions dynamics and the interactions between molecules. Of particular interest to physical chemistry of LCPs are the far-IRactive degrees of freedom, which correspond to soft, large-amplitude LCP vibration modes directly involved in the molecular dynamics. These properties make $\mathrm{THz}$ spectroscopy an ideal tool for discriminating between polymorphic forms of fibers and granules Vectra A950. It is known that the difference between the molecular chain orientation in fibers and in the bulk of granules is highly sensitive to details of their thermal prehistory, i.e., it depends on the chain connectivity (intermolecular interactions) and on the dynamics of large-amplitude vibrational and torsional modes in LCP [16].

Far-IR spectra of Vectra A950 fibers and granules are shown in Figure 2. Comparison of these spectra indicates that they differ most significantly just at low frequencies, To identify the absorption bands in the farIR spectrum of Vectra A950 we used experimental and calculated far-IR spectra of hydroxy-benzoic acid [17] and hydroxy-naphthoic acid [18], which enter into the composition of this copolymer. The tentative assignment of the absorption bands in the far-IR spectrum of Vectra A-950, made on the basis of these 
Table 1: The Characteristics of the Absorption Bands in Far-IR Spectra Vectra A950

\begin{tabular}{|c|c|c|c|}
\hline Vibrational modes Vectra A950 (cm-1) & Approximate Description & $\mathbf{R}_{\mathbf{G}}$ & $\mathbf{R}_{\mathbf{F}}$ \\
\hline 69 & $\mathrm{~N}$ - ring libration & 0.75 & 0.95 \\
\hline 96 & B- ring libration & 0.95 & 1.15 \\
\hline 127 & Poley absorption + COO torsion & 0.45 & 1.55 \\
\hline 143 & v $10 \mathrm{~b}$ B- ring & 1.65 & 1.55 \\
\hline 169 & `Batterfly` N- ring & 1.50 & 1.55 \\
\hline 235 & CCC def. & 1.42 & 1.33 \\
\hline 262 & COC torsion & 1.55 & 1.43 \\
\hline 338 & $v B_{1 u} N$ - ring & 1.55 & 1.43 \\
\hline 355 & `Envelope flap` N-ring & 1.65 & 1.55 \\
\hline 407 & v 16a B-ring & 1.20 & 1.25 \\
\hline 475 & $\mathrm{~N}$-ring def. $\left(\mathrm{B}_{3 \mathrm{u}}\right)$ & 1.25 & 1.33 \\
\hline
\end{tabular}

Symbols: F-fibers; G-granules; N-naphthalene; B-benzene; def.- deformation.

data, and the dichroic ratio of these bands, obtained from polarized measurements on the fibers and granules Vectra A950, are presented in the Table 1.

Comparison the dichroic ratio of the absorption bands in the spectra of the granules $(R G=A \| G / A \perp G)$ and fibers $(R F=A \| F / A \perp F)$ shows that the values of $R F$ and $R G$ are not very different from each other. This is so except in the lowest-frequency bands, characterizing torsional vibrations (libration) of mesogens (phenylene and naphthalene rings) in the copolymer. Especially different are the values of $R G$ and RF (and, therefore, the orientation order parameters), obtained for the absorption band at 127 $\mathrm{cm}^{-1}$, which is due to Poley-type absorption [19, 20]. Polarized far-IR spectra of Vectra A950 fibers and granules in the range $127 \mathrm{~cm}^{-1}$ are shown in Figure 3 .

The Poley-type absorption, which is now well known to be due to molecular torsional oscillations (librations), has been observed previously in far-IR spectra of

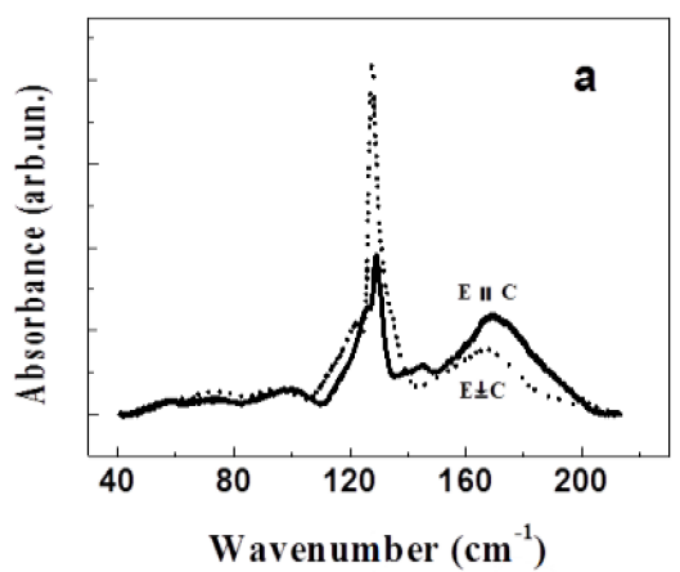

liquid- crystals only for nematics, such as 4methoxybenzylidene-4- butylaniline (MBBA). The Poley absorption in these spectra has the same half-width and intensity of lines as those in spectra of typical polar liquids and the peak of this absorption should be expected at $v=50 \mathrm{~cm}^{-1}$ in MBBA. That it appeared centered near $130 \mathrm{~cm}^{-1}$, having intensity substantially greater than expected for a difference tone or an intramolecular torsional mode in the MBBA molecule, leads the authors to the conclusion about the specifics of the librational movement in the LC. They believe that the difference between the calculated value of the librational frequency and the experimental frequency is a consequence of the deeper and narrower energy well in which the lath-like molecules librate. Indeed, X-ray evidence implies that around each molecule MBBA there is approximately a hexagonal array of parallel molecules [21]. Such an environment provides a steeper potential well with respect to angular displacements around the long axis of the mesogen (i.e. with a smaller moment of inertia and less hindered

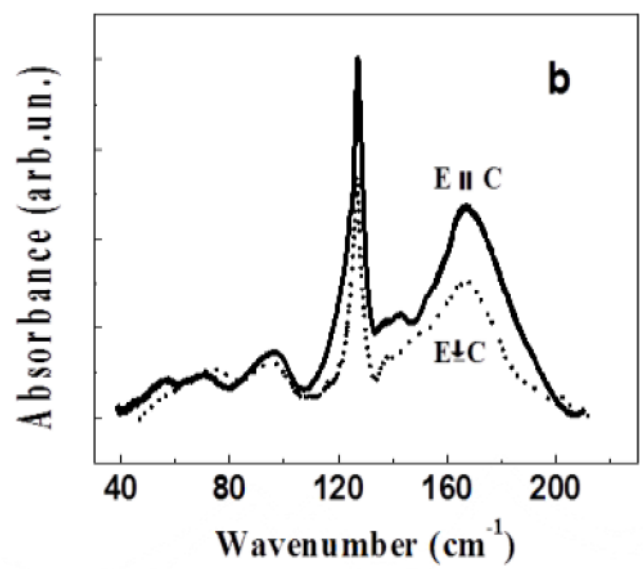

Figure 3: Polarized far- IR absorption spectra Vectra A950: granules (a), the fibers (b) in the range $30-250 \mathrm{~cm}^{-1}$. 
rotation) and thus a higher value of the librational frequency.

These findings also are valid in the present case. It should only be added that in the formation of the spectrum at these frequencies can participate and intramolecular mobility, such as the torsional vibrations of the COO group, which connects mesogens in the copolymer.

A detailed discussion of the assignment of the absorption band at $127 \mathrm{~cm}^{-1}$ in the far-IR spectrum of Vectra A950 was needed because just this band is the most sensitive to the orientation ordering of copolymer. The dichroic ratio for the majority bands in the far-IR spectrum (see Table 1) and for the bands in the mid-IR (see Figure 1) spectrum of Vectra A950 granules practically does not differ from those of the fibers. So, the dichroic ratio for the band of stretching vibrations of a carbonyl group at $1727 \mathrm{~cm}^{-1}$ in the spectrum of granules $R G=0.85$, and that in the spectrum fibers, $\mathrm{RF}=1.3$, which are close to each other, whereas the corresponding values for the band at $127 \mathrm{~cm}^{-1}$ in the far-IR spectra, $R G=0.45$ and $R F=1.55$ and these values differ by more than a factor of 3 . As a result, the parameters of the orientation order for the granules and fibers of the copolymers, derived from of the mid-IR spectra, were close to zero, which does not allow distinguishing the orientation ordering in granules Vectra A 950 from the orientation ordering in fibers Vectra A 950. While the parameters of the orientation order, obtained from the dichroic ratio for the libration band at $127 \mathrm{~cm}^{-1}$ in the spectra of the granules and fibers $\left(S_{G}=-0.22\right.$ и $S_{F}=0.16$, respectively), clearly indicate a higher orientation of chains in the draw direction in fibers of copolymer, in contrast to its granules. This result suggests that the orientation state of the LCP type Vectra A 950, is largely determined by the local dynamics of polymer chains, such as the torsional mobility of aromatic mesogens.

\section{CONCLUSIONS}

The polarized far-IR spectroscopy was successfully applied to characterize the molecular orientation of the granules and fibers of a completely aromatic thermotropic liquid-crystal copolymer Vectra A950. It was found that the absorption bands arising from the manifestation of external degrees of freedom of mesogens (benzene and naphthalene rings) and, their libration or torsions are the most sensitive to the orientational ordering in the LCP Vectra A950.The results suggest the possibility of carrying out an independent evaluation of the spectroscopic parameters reflecting the properties of intermolecular interactions in oriented LCP from their low-frequency spectra. It demonstrated that this frequency range is an extremely important spectral range for polymer science.

\section{ACKNOWLEDGMENTS}

The authors are grateful to A.Yu. Bilibin for the polymers he presented. The study was supported by the Russian Foundation for Basic Research, project no. 93-03-32624.

\section{REFERENCES}

[1] Thakur VK, Kessler MR, Eds. Liquid Crystal Polymers. Processing and Applications. Springer International Publishing: Switzerland 2015.

[2] Liu D, Broer DJ. Liquid Crystal Polymer Networks: Preparation, Properties, and Applications of Films with Patterned Molecular Alignment. Langmuir 2014; 30: 1349910 https://doi.org/10.1021/la500454d

[3] Shengtong S, Hui T, Peiyi W. FT-IR Characterization of Liquid Crystalline Polymers. Progress in Chemistry 2009; 21: 182-199.

[4] Savitsky AV, Gorshkova IA, Kober KJ. Orientational Ordering in Fibers Made of Wholly Aromatic Main Chain LC Polyesters. J Macromol Sci-Phys 1998; B37: 119-10. https://doi.org/10.1080/00222349808220458

[5] Wiberg G, Hillborg H, Gedde UW. Assessment of Development in a Sheared Thermotropic Liquid Crystalline Copolyester. Polym Eng Sci 1998; 38: 1278-7. https://doi.org/10.1002/pen.10298

[6] Jin X, Chung T-S. Thermal Decomposition Behavior of MainChain Thermotropic Liquid Crystalline Polymers, Vectra A950, B-950, and Xydar SPT-900. J Appl Polym Sci 1999; 73: 2195-12.

https://doi.org/10.1002/(SICI)10974628(19990912)73:11<2195::AID-APP17>3.0.CO;2-3

[7] De Gennes PG. Reptation of a Polymer Chain in the Presence of Fixed Obstacles. J Chem Phys 1971; 55: 572-8. https://doi.org/10.1063/1.1675789

[8] Vieweg N, Celik MA, Zakel S, Gupta V, Frenking G, Koch M Terahertz Absorption of Nematic Liquid Crystals. J Infrared Milli Terahz Waves 2014; 35: 478-7. https://doi.org/10.1007/s10762-014-0062-3

[9] Savitsky AV, Bilibin AYu, Gorshkova IA. On the Problem of Obtaining High-Strength Fibers from Liquid-Crystalline Polymers. Vysokomol Soed 1992; A34: 722-5.

[10] Egorov EA, Zhizhenkov VV. Molecular Dynamics and Hardening of Liquid Crystal Polymers. Phys Solid State 2005; 47: 914-6.

https://doi.org/10.1134/1.1924859

[11] Beekmans F, Posthuma de Boer A. Determination of Orientation in Thermotropic Liquid Crystalline Polymer Films by Spectrographic Measurement of the Birefringence. Macromolecules 1996; 29: 8726-7. https://doi.org/10.1021/ma960860t

[12] Abdullin SN, Furer VL, Bilibin AYu. Analysis of IR -spectra of Thermotropic Liquid-Crystalline Aromatic Polyesters. Vysokomol Soed 1990; B32: 701-6. 
[13] Tai-Shung C, Xing J. Studies on the Phase Tranition and Thermal Stability of Xydar and Zenit Series Liquid Crystalline Polymer. Polym Eng Sci 2000; 40: 841-15. https://doi.org/10.1002/pen.11213

[14] Vieweg N, Deninger A. Terahertz Waves and Liquid Crystals: Prospects and Challenges. Proc of SPIE 2013; 8642: 86420K-1-12.

https://doi.org/10.1117/12.2013315

[15] Rutz F, Hasek T, Koch M, Richter H, Ewert U. Terahertz birefringence of liquid crystal polymers. Appl Phys Lett 2006; 89: 221911-8.

https://doi.org/10.1063/1.2397564

[16] Evans M, Davies M, Larkin I. Molecular Motion and Molecular Interaction in the Nematic Isotropic Phases of a Liquid Crystal Compound. J Chem Soc Faraday Trans 2 1973; 69: 1011-11.

https://doi.org/10.1039/f29736901011

[17] Takahashi M, Kawazoe $Y$, Ishikawa $Y$, Ito $H$. Interpretation of temperature-dependent low frequency vibrational spectrum of solid-state benzoic acid dimer. Phys Lett 2009; 479: 211-6. https://doi.org/10.1016/j.cplett.2009.08.017
[18] Ponseca S, Estacio E, Murakami H, Sarukura N, Pobre R, Tominaga K, Nishizawa J, et al. Experimental and calculated terahertz spectra of naphthalene and 1,4dihydroxynaphthalene in the $0.5-6$ terahertz region. J Phys Conference Ser 2008; 112(part 4): 042073-4. https://doi.org/10.1088/1742-6596/112/4/042073

[19] Evans M, Moscicki JK. The Poley Absorption in Liquid Crystals. J Molec Liquids 1986; 32: 149-11. https://doi.org/10.1016/0167-7322(86)80020-4

[20] Bershtein VA, Ryzhov VA. Far Infrared Spectroscopy of Polymers. Adv Polym Sci 1994; 114: 43-78. https://doi.org/10.1007/BFb0008694

[21] Vries A. X-Ray Photographic Studies of Liquid Crystals: II. Apparent Molecular Length and Thickness in Three Phases of Ethyl-p-ethoxybenzal-p-aminobenzoate. Mol Cryst Liquid Cryst 1970; 11: 361-5. https://doi.org/10.1080/15421407008083528 